\title{
Changes in the Electronic Structure of Tetra-Tert-Butyl Copper Phthalocyanine Film by Ultraviolet-Ozone Treatment
}

\author{
Received 4 January, 2021; accepted 26 January, 2021 \\ Wonsik Kim, Seungsun Choi, Woojin Shin, Jaewon Oh, Mee-Yi Ryu, and Hyunbok Lee* \\ Department of Physics and Institute for Accelerator Science, Kangwon National University, Chuncheon 24341 , \\ Republic of Korea
}

\author{
*Corresponding author E-mail: hyunbok@kangwon.ac.kr
}

\begin{abstract}
Ultraviolet-ozone (UVO) treatment modifies the surface properties of film for use in thin-film optoelectronics. In particular, the hydrophobic nature of the surface of organic films can be changed to a hydrophilic nature, thereby enabling a multilayer structure using a solution process. However, the electronic structure of organic films with UVO treatment has not been investigated in detail. In this study, the changes in the electronic structure of tetra-tert-butyl copper phthalocyanine (ttb-CuPc) films by UVO treatment were explored using UV-vis absorption spectroscopy and X-ray photoelectron spectroscopy (XPS). The ttb-CuPc film was fabricated by spin coating, and various UVO treatment times were applied. As the UVO treatment time increased, the absorption intensity of the ttb-CuPc films exponentially decayed. In the XPS spectra, the oxidation process was observed in two stages (macrocyclic isoindole units followed by peripheral ttb units). Upon oxidation, while the $\mathrm{C}$ and $\mathrm{N}$ atoms in the ligands exhibited an electron-donating characteristic, the central $\mathrm{Cu}$ atom showed an electron-withdrawing nature. Such an altered charge distribution on the ttb-CuPc molecule can significantly influence charge transport in optoelectronic devices.
\end{abstract}

Keywords: X-ray photoelectron spectroscopy, Tetra-tert-butyl copper phthalocyanine, Ultraviolet-ozone treatment, Oxidation, UV-vis absorption spectroscopy

\section{Introduction}

In recent decades, organic semiconductors have been used in various optoelectronic devices, including light-emitting diodes and solar cells [1]. In these applications, organic semiconductors serve not only as a light-emitting or absorbing layer, but also as a charge transport layer [2]. In many thin-film optoelectronic devices, a multilayered structure is chosen to improve the device performance. However, when the solution process is employed, the fabrication of the multilayered structure often suffers from the hydrophobicity of the underlying layer. Although the solution process is cost efficient because it does not require the use of expensive vacuum facilities, it can limit the selection range of the device architecture. Thus, developing an efficient method for fabricating a multilayer structure using the solution process is important for realizing low-cost devices with high efficiency.

One of the most renowned methods of rendering the surface hydrophilic is ultraviolet-ozone (UVO) treatment. UVO treatment removes carbon-based contaminants and generates an oxygen-rich surface [3-6]. As a result, the second layer can be deposited onto such a modified surface using a solution process (e.g., spin coating). However, severe UVO treatment significantly alters the surface composition, which may deteriorate the charge transport properties and eventually degrade the device performance. Thus, determining the optimum duration of UVO treatment is important to obtaining the best device performance. To this end, a fundamental study on the changes in the electronic structure of an organic film under UVO treatment is necessary [7]. However, only a few studies have been reported on this issue. Thus, the impact of UVO treatment should be explored for various organic semiconductors.
Tetra-tert-butyl copper phthalocyanine (ttb-CuPc) is a molecule that consists of copper phthalocyanine $(\mathrm{CuPc})$ and peripheral tetratert-butyl (ttb) units. CuPc is a well-known p-type organic semiconductor with low ionization energy [8-10]; however, it does not have satisfactory solubility. Nevertheless, the attachment of ttb units increases the solubility, thereby enabling the film to be deposited by the solution process. Such a ttb-CuPc film works as a sensitive layer in gas sensors and as a hole transport layer in solar cells [11,12]. For improved use of ttb-CuPc, UVO treatment may be useful.

With consideration of the above background, we investigated the electronic structure of ttb-CuPc films with UVO treatment. The changes in the light-absorbing ability of the films were measured by UV-vis absorption spectroscopy. To determine the energy-level shift and chemical interaction, X-ray photoelectron spectroscopy (XPS) was employed. The films were prepared with various UVO treatment times, and their UV-vis absorption and XPS spectra were obtained. The charge distribution on an oxidized ttb-CuPc molecule was examined using the curve fittings of the XPS spectra.

\section{Experimental details}

Indium tin oxide (ITO)-coated glass substrates were cleaned by ultrasonication in methanol, deionized (DI) water, detergent, and acetone. Subsequently, the ITO substrates were dried using $\mathrm{N}_{2}$ gas flow and then treated with UVO at $100{ }^{\circ} \mathrm{C}$ for $15 \mathrm{~min}$ using a PSDP-UV4T UVO cleaner (Novascan Technology Inc., Iowa United States). The ttb-CuPc powder was purchased from Sigma-Aldrich and it was used as received. The ttb-CuPc powder was dissolved in toluene (purity 
(a)

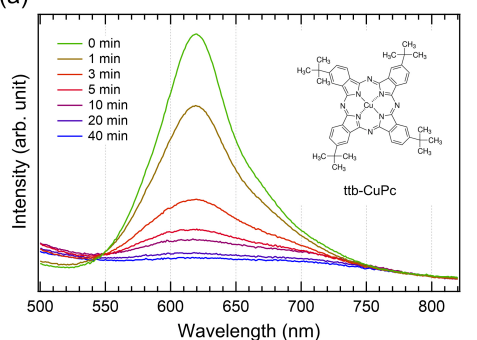

(b)

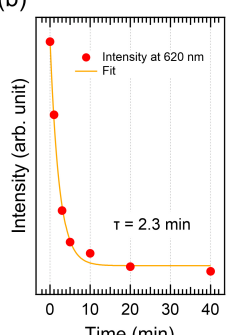

(a)

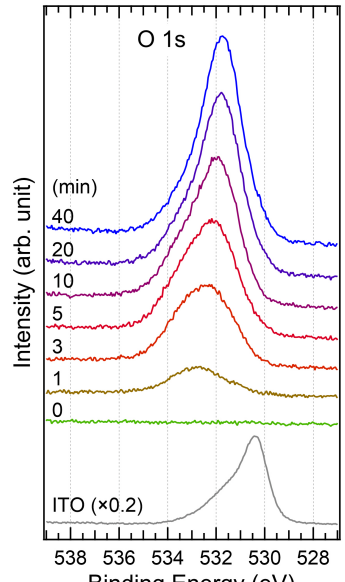

(b)

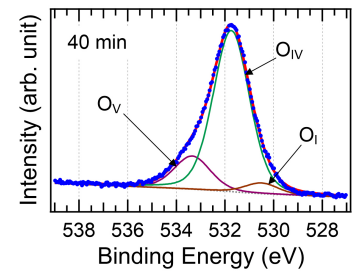

(c)

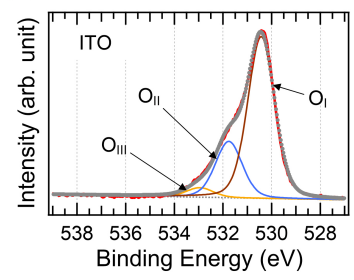

Figure 1. (a) UV-vis absorption spectra of ttb-CuPc films with various UVO treatment times $(0,1,3,5,10,20$, and $40 \mathrm{~min})$ (inset: ttb-CuPc chemical structure) and (b) intensity decay trend at $620 \mathrm{~nm}$.

$\geq 99.9 \%$, Sigma-Aldrich, St. Louis, Missouri, United States) at a concentration of $4 \mathrm{mg} \mathrm{mL}^{-1}$, and it was used after stirring overnight. The $\mathrm{ttb}-\mathrm{CuPc}$ film was deposited by spin coating at a spin rate of $2000 \mathrm{rpm}$ for $60 \mathrm{~s}$. Subsequently, the deposited film was annealed at $150{ }^{\circ} \mathrm{C}$ for $10 \mathrm{~min}$ to remove residual solvent. The thickness of the ttb-CuPc film was estimated to be approximately $20 \mathrm{~nm}$ using an Alpha-Step D500 surface profiler (KLA-Tencor, Milpitas, California, United States). The films were treated with UVO for various durations using a UVO cleaner at room temperature.

The UV-vis absorption spectra of the ttb-CuPc films with UVO treatment were measured using an Ubi-490 spectrometer (MicroDigital Co., Ltd., Gyeonggi-do, South Korea). The XPS spectra were measured using the K-Alpha ${ }^{+}$system (Thermo Fisher Scientific Inc., Waltham, Massachusetts, United States) and an Al K $\alpha(h v=1486.6$ eV) X-ray source. The diameter of the analysis area in XPS is $400 \mu \mathrm{m}$. The XPS spectra were collected with $20 \mathrm{eV}$ pass energy. An electron flood gun was not used.

\section{Results and discussion}

Figure 1 shows (a) the UV-vis absorption spectra of ttb-CuPc films with various UVO treatment times $(0,1,3,5,10,20$, and $40 \mathrm{~min})$ and (b) the decay trend of the spectral intensity of their maximum peak at $620 \mathrm{~nm}$. The chemical structure of ttb-CuPc is shown in the inset. In Fig. 1(a), the main absorption features are observed in the range of $550-750 \mathrm{~nm}$, and the maximum peak is observed at $620 \mathrm{~nm}$. The measured spectrum of the pristine ttb-CuPc film matches well with those in the literature [12-14]. Because the spectrum of the long-wavelength region is highly dispersive, it is not easy to accurately determine the absorption onset. However, the onset may occur in the approximate range of $750-800 \mathrm{~nm}$. Thus, the optical gap of the ttb-CuPc film is estimated to be approximately $1.6 \mathrm{eV}$.

As the UVO treatment is performed, the spectral intensity monotonically decreases. At the final step, the UVO treatment time of 40 min, the absorption features are hardly observed. This decrease in the spectral intensity is attributed to both the destruction of the conjugated system and the decrease in the film thickness [7]. In Fig. 1(b), the spectral intensity significantly decreases in several minutes. The intensity of the UVO-treated ttb-CuPc film for $5 \mathrm{~min}$ is only approximately $20 \%$ of that of the pristine film. The decay trend can be roughly fitted with a single exponential function as follows:

$$
y=y_{0}+A \exp \left(-\frac{t}{\tau}\right)
$$

where $y_{0}$ and $A$ are constants and $\tau$ is the time constant. As a result, $\tau$ is calculated to be $2.3 \mathrm{~min}$. This value is much smaller than those of the polymeric semiconductors in our recent study [7]. This could be attributed to the different inter- and intra-molecular interactions. Nonetheless, further study is necessary to determine the exact origin of such difference.

Figure 2. (a) XPS O 1s core-level spectra of ITO and ttb-CuPC films with various UVO treatment times $(0,1,3,5,10,20$, and $40 \mathrm{~min})$ and fittings of (b) ttb-CuPc films with a UVO treatment time of 40 min and (c) ITO. (a)

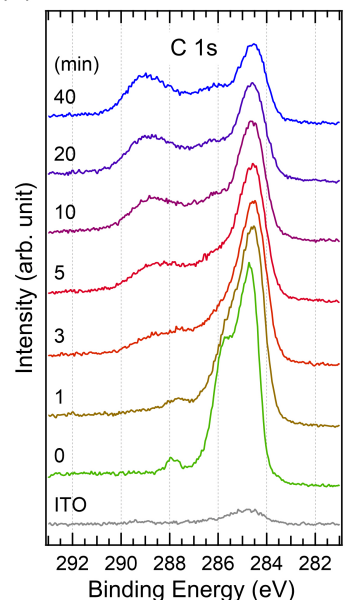

(b)

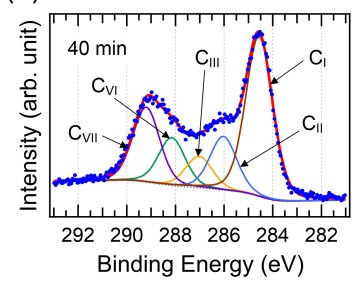

(c)

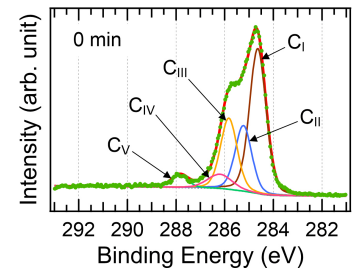

Figure 3. (a) XPS C 1s core-level spectra of ITO and ttb-CuPC films with various UVO treatment times $(0,1,3,5,10,20$, and $40 \mathrm{~min})$ and fittings of ttb-CuPc films with UVO treatment times of (b) 40 and (c) 0 min.

Figure 2 shows (a) the XPS O 1s core-level spectra of ITO and ttbCuPc films with various UVO treatment times $(0,1,3,5,10,20$, and 40 $\mathrm{min}$ ) and the spectral fittings of (b) the ttb-CuPc film with the UVO treatment time of $40 \mathrm{~min}$ and (c) ITO. In Fig. 2(a), the bottommost spectrum represents the minified ITO $(\times 0.2)$. As the ttb-CuPc film is deposited $(0 \mathrm{~min})$, the $\mathrm{O} 1 \mathrm{~s}$ features from the ITO are no longer detectable. This indicates that the thickness of the ttb-CuPc film is larger than the XPS probing depth (approximately $10 \mathrm{~nm}$ ), which agrees well with the film thickness measured by using the surface profiler.

However, as the UVO treatment was performed, the new oxidation features emerged. As the UVO treatment time increased, the intensity of the oxidation features increased, indicating an increase in the degree of oxidation. In addition, the peak position seemingly shifted toward a lower binding energy. With the UVO treatment time of $1 \mathrm{~min}$, the maximum intensity position was observed at $532.8 \mathrm{eV}$, whereas with the UVO treatment time of $40 \mathrm{~min}$, it was observed at $531.7 \mathrm{eV}$. Nevertheless, considering the spectral shapes for the UVO treatment times of 3 and $5 \mathrm{~min}$, such oxidation features could not be fitted by using a single curve. Thus, they were composed of two different components, and the peak at the lower binding energy became higher as the UVO 
(a)

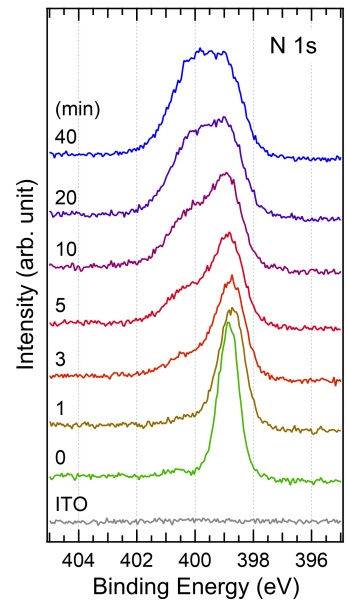

(b)

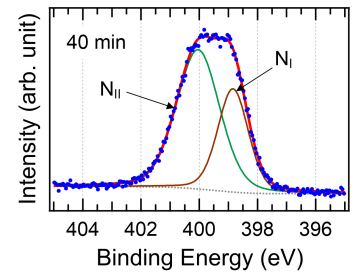

(c)

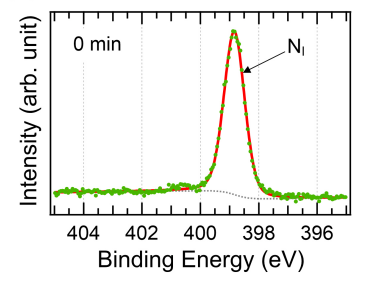

Figure 4. (a) XPS N 1s core-level spectra of ITO and ttb-CUPC films with various UVO treatment times $(0,1,3,5,10,20$, and $40 \mathrm{~min})$ and fittings of ttb-CuPc films with UVO treatment times of (b) 40 and (c) 0 min.

treatment time increased. In other words, the energy shift in the maximum intensity position was due to the sequential oxidation process in two stages. First, the oxidation peak at the higher binding energy was dominant. Subsequently, the oxidation peak at the lower binding energy was dominant. The oxidation process of an organic semiconductor can be varied by the type of light and oxygen exposure $[15,16]$.

To analyze the oxidation mechanism, we conducted fittings using a Voigt function, as shown in Figs.2(b) and 2(c). In Fig. 2(c), the ITO is fitted by using three components-the $\mathrm{O}_{\text {I }}$ peak at $530.4 \mathrm{eV}, \mathrm{O}_{\text {II }}$ peak at $531.8 \mathrm{eV}$, and $\mathrm{O}_{\text {III }}$ peak at $533.0 \mathrm{eV}$-originating from the lattice oxygen, oxygen vacancies, and surface contamination, respectively. These are in good agreement with the literature [17, 18]. In Fig. 2(b), for the UVO treatment time of $40 \mathrm{~min}$, which shows the highest degree of oxidation in this study, the spectrum is fitted using three components. First, the substrate peak $\left(\mathrm{O}_{\mathrm{I}}\right)$ appears again. This is due to a decrease in the film thickness. In the XPS spectra of the In $3 \mathrm{~d}$ region (not shown here), the In $3 d_{5 / 2}$ and $3 d_{3 / 2}$ peaks are hardly observed in the pristine ttb-CuPc film. However, they are observed again with the UVO treatment time of $40 \mathrm{~min}$. This finding agrees well with previous reports demonstrating that the UVO treatment etches the organic film surface $[7,16]$. From the In $3 d$ peak attenuation, the thickness of the ttb-CuPc with the UVO treatment of $40 \mathrm{~min}$ is estimated to be approximately $9 \mathrm{~nm}$, which is thinner than half of the original thickness. In Fig. 2(b), only the major lattice oxygen peak is considered for simplicity. The $\mathrm{O}_{\mathrm{IV}}$ peak at $531.7 \mathrm{eV}$ is surmised to originate from the $\mathrm{C}-\mathrm{O}$ bond formed on the peripheral ttb units, whereas the $\mathrm{O}_{\mathrm{V}}$ peak at 533.4 is believed to occur owing to the $\mathrm{N}-\mathrm{O}$ bond formed on the macrocyclic isoindole units, respectively. Thus, oxidation mainly occurs first on the macrocyclic isoindole units and then on the peripheral ttb units.

Figure 3 shows (a) the XPS C 1s core-level spectra of the ttb-CuPc films with various UVO treatment times $(0,1,3,5,10,20$, and $40 \mathrm{~min})$ and the spectral fittings with UVO treatment times of (b) 40 and (c) 0 min. In Fig. 3(a), the bottommost spectrum shows that the ITO has slight contamination. As the ttb-CuPc film is deposited, the characteristic isoindole features of metal phthalocyanine $(\mathrm{MePc})$ and aliphatic features of the ttb units are simultaneously observed. As the UVO treatment is performed, new peaks emerge at higher binding energies than those of the original peaks. As the UVO treatment time increase, the intensities of the oxidation peaks increase, whereas those of the original peaks decrease, showing an increase in the degree of oxidation.

To observe the oxidation features in detail, fittings were conducted,

(a)

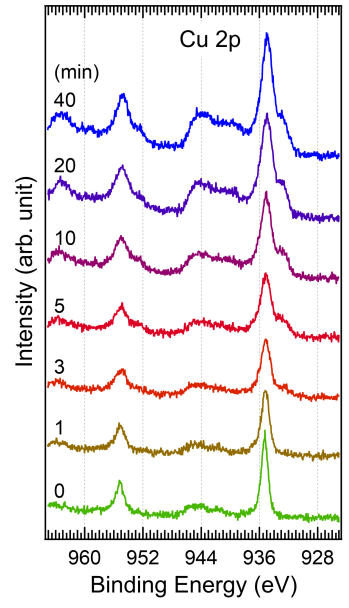

(b)

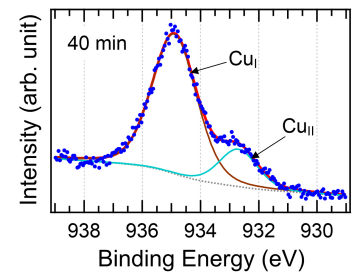

(c)

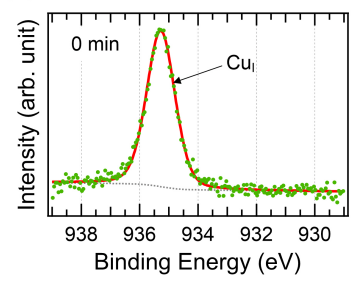

Figure 5. (a) XPS Cu 2p core-level spectra of ttb-CuPc films with various UVO treatment times $(0,1,3,5,10,20$, and $40 \mathrm{~min})$ and fittings of ttb-CuPc films with UVO treatment times of (b) 40 and (c) 0 min.

as shown in Figs.3(b) and 3(c). In Fig. 3(c), the $C$ 1s spectrum of the pristine ttb-CuPc film is fitted with five components. The $\mathrm{C}_{\mathrm{I}}$ and $\mathrm{C}_{\mathrm{III}}$ peaks at 284.6 and $285.8 \mathrm{eV}$ correspond to the aromatic $\mathrm{C}-\mathrm{C}$ and pyrrole C-N bonds commonly observed in MePc, respectively $[19,20]$. The $\mathrm{C}_{\mathrm{IV}}$ peak at $286.2 \mathrm{eV}$ and the $\mathrm{C}_{\mathrm{V}}$ peak at $287.8 \mathrm{eV}$ are their shakeup peaks from the $\pi-\pi^{*}$ transition. The $\mathrm{C}_{\text {II }}$ peak at $285.2 \mathrm{eV}$ is the aliphatic $\mathrm{C}-\mathrm{C}$ bond in the ttb units. According to the ttb-CuPc chemical formula, the intensity ratio of $\mathrm{C}_{\mathrm{I}}: \mathrm{C}_{\mathrm{II}}: \mathrm{C}_{\mathrm{III}}$ should be $3: 2: 1$. However, the measured ratio is approximately $2.8: 1.3: 1.4$. This discrepancy was similarly observed in a tetra-tert-butyl magnesium phthalocyanine (ttb-MgPc) film [21]. The atomic bonding ratio of the ttb-MgPc film was significantly varied by the choice of the photon energy and substrate. The nominal stoichiometry could be obtained with high photon energy and a single-crystal substrate. This implies that the molecular orientation can affect the atomic ratio calculated by the XPS intensities.

In Fig. 3(b), as the UVO treatment is performed, the $\mathrm{C}_{\mathrm{II}}$ and $\mathrm{C}_{\mathrm{III}}$ peaks shift toward higher binding energies. The peak positions of $\mathrm{C}_{\mathrm{II}}$ and $\mathrm{C}_{\mathrm{III}}$ with the UVO treatment time of $40 \mathrm{~min}$ are estimated to be 286.0 and $287.0 \mathrm{eV}$, respectively. In addition, the full width at half maximum (FWHM) of these peaks considerably increases from to 0.8 to $1.3 \mathrm{eV}$. The $\mathrm{C}_{\mathrm{VI}}$ and $\mathrm{C}_{\mathrm{VII}}$ peaks formed by oxidation are observed at 288.2 and $289.2 \mathrm{eV}$, originating from the $\mathrm{C}-\mathrm{O}-\mathrm{H}$ and $\mathrm{C}-\mathrm{N}-\mathrm{O}$ bonds, respectively. For simplicity, the weak shake-up peaks were omitted in the fitting. These spectral changes show that oxidation occurs in various atomic positions on ttb-CuPc. These changes demonstrate a similar trend as those observed in the XPS O 1s spectra. However, the XPS C 1s peaks do not show the large shift in the energy position observed in the $\mathrm{O}$ 1s peaks. This supports the finding that the peak shift in the $\mathrm{O}$ 1s spectra is not due to the band bending; rather, it is caused by the sequential oxidation process in two stages.

Figure 4 shows (a) the XPS N 1s core-level spectra of the ttb-CuPc films with various UVO treatment times $(0,1,3,5,10,20$, and $40 \mathrm{~min})$ and the spectral fittings with UVO treatment times of (b) 40 and (c) 0 min. In Fig. 4(a), the bottommost ITO spectrum shows no $\mathrm{N} 1 \mathrm{~s}$ signal. However, the typical N 1s features of MePc appear as the ttb$\mathrm{CuPc}$ film is deposited. As UVO treatment is performed, the new peak emerges at its higher binding energy. As the UVO treatment time is increased, the intensity of the original peak decreases, whereas that of the oxidation peak gradually increases.

In Fig. 4(c), the $\mathrm{N}_{\mathrm{I}}$ peak due to the isoindole units is observed at $398.8 \mathrm{eV}$. For simplicity, the $\mathrm{N} 1 \mathrm{~s}$ peak is fitted with a single component without distinguishing between the $\mathrm{C}-\mathrm{N}=\mathrm{C}$ and $\mathrm{C}-\mathrm{N}(-\mathrm{Cu})=\mathrm{C}$ 
bonds. With the UVO treatment time of $40 \mathrm{~min}$, the $\mathrm{N}_{\mathrm{II}}$ peak due to oxidation emerges at $400.0 \mathrm{eV}$, and its intensity is higher than that of the $\mathrm{N}_{\mathrm{I}}$ peak. The $\mathrm{N}_{\mathrm{I}}$ peak broadens on account of the UVO treatment, and its FWHM increases from 0.84 to $1.24 \mathrm{eV}$. Thus, the changes in the chemical states occur on the whole ttb-CuPc molecule.

Figure 5 shows (a) the XPS $\mathrm{Cu} 2 \mathrm{p}$ core-level spectra of ttb-CuPc films with various UVO treatment times $(0,1,3,5,10,20$, and 40 $\mathrm{min}$ ) and the spectral fittings of the $\mathrm{Cu} 2 \mathrm{p}_{3 / 2}$ peak with UVO treatment times of (b) 40 and (c) 0 min. In Fig. 5(a), the two $\mathrm{Cu} 2 \mathrm{p}_{3 / 2}$ and $2 \mathrm{p}_{1 / 2}$ peaks due to the spin-orbit splitting are observed at $935.3 \mathrm{eV}$ and 955.2 $\mathrm{eV}$. The broad satellite features of the $\mathrm{Cu} 2 \mathrm{p}_{3 / 2}$ peak are observed in the range of $940-948 \mathrm{eV} \mathrm{[22].} \mathrm{As} \mathrm{the} \mathrm{UVO} \mathrm{treatment} \mathrm{is} \mathrm{conducted,}$ the original $\mathrm{Cu} 2 \mathrm{p}$ peaks broaden. In addition, new peaks emerge at lower binding energies. As the UVO treatment time is increased, the intensities of the new peaks increase.

In Fig. 5(c), the $\mathrm{Cu} 2 \mathrm{p}_{3 / 2}$ peak of the pristine ttb-CuPc film $\left(\mathrm{Cu}_{\mathrm{I}}\right)$ is fitted with a single curve. In Fig. 5(b), for the UVO treatment time of $40 \mathrm{~min}$, the new $\mathrm{Cu} 2 \mathrm{p}_{3 / 2}$ peak $\left(\mathrm{Cu}_{\mathrm{II}}\right)$ formed by oxidation is observed at $932.7 \mathrm{eV}$. The FWHM of the $\mathrm{Cu}_{\mathrm{I}}$ peak significantly increases from 1.15 to $1.81 \mathrm{eV}$. Meanwhile, the new $\mathrm{C} 1 \mathrm{~s}$ and $\mathrm{N} 1 \mathrm{~s}$ peaks appear at a higher binding energy than those of the original peaks, and the new $\mathrm{Cu}_{\mathrm{II}}$ peak (and corresponding new $\mathrm{Cu} 2 \mathrm{p}_{1 / 2}$ peak) is observed at a lower binding energy than that of the original peak. This indicates that the $\mathrm{C}$ and $\mathrm{N}$ atoms demonstrate the electron-donating nature; however, the $\mathrm{Cu}$ atom shows the electron-withdrawing nature on the oxidized ttb-CuPc molecule. Thus, the electron densities in the $\mathrm{C}$ and $\mathrm{N}$ atoms in the ligands would decrease, whereas that in the central $\mathrm{Cu}$ atom would increase. Such a charge redistribution on the whole molecule impact both the carrier mobility in the ttb-CuPc film and the energy-level alignment with the adjacent layers in the multilayer system.

\section{Conclusion}

In this study, we investigated the changes in the electronic structure of ttb-CuPc films due to UVO treatment. In the UV-vis absorption spectra, the absorption intensity of the ttb-CuPc films decayed exponentially as the UVO treatment time increased. In the XPS spectra, new oxidation peaks were observed in all core-level regions with UVO treatment, and their intensities increased as the degree of oxidation increased. The evolution of the $\mathrm{C} 1 \mathrm{~s}$ spectra in accordance with an increasing UVO treatment time showed that oxidation occurred via two sequential stages. The new $\mathrm{C} 1 \mathrm{~s}$ and $\mathrm{N} 1 \mathrm{~s}$ peaks emerged at higher binding energies than those of the original peaks. However, new $\mathrm{Cu}$ $2 p$ peaks emerged at lower binding energies than those of the original peaks. This indicates that the charge distribution on a molecule is significantly altered by oxidation. These electronic structures that were changed by UVO treatment could have a critical effect on the device performance. Thus, the optimum duration of UVO treatment should be carefully chosen while considering such properties.

\section{Acknowledgements}

This study was supported by the National Research Foundation of Korea (NRF-2018R1D1A1B07051050 and 2018R1A6A1A03025582),
Korea Foundation for the Advancement of Science \& Creativity (KOFAC), and Supporting Business for College Innovation from Kangwon National University. The XPS measurements were performed with the instrument at the Central Laboratory of Kangwon National University.

\section{References}

[1] W. Brütting, Physics of Organic Semiconductors (Wiley-VCH, Weinheim, 2005)

[2] F. So, Organic Electronics: Materials, Processing, Devices and Applications (CRC Press, Florida, 2010).

[3] K. Sugiyama, H. Ishii, Y. Ouchi, and K. Seki, J. Appl. Phys. 87, $295(2000)$

[4] W. Song, S. K. So, D. Wang, Y. Qiu, and L. Cao, Appl. Surf. Sci. $177,158(2001)$

[5] A. T. Oluwabi, D. Gaspar, A. Katerski, A. Mere, M. Krunks, L. Pereira, and I. O. Acik, Materials 13, 6 (2020).

[6] S. J. Han, J. H. Kim, J. W. Kim, C. K. Min, S. H. Hong, D. H. Kim, K. H. Baek, G. H. Kim, L. M. Do, and Y. Park, J. Appl. Phys. 104, 013715 (2008).

[7] S. Choi, W. Kim, W. Shin, J. Oh, S. Jin, Y. M. Jung, M. Y. Ryu, and H. Lee, Curr. Appl. Phys. 20, 1359 (2020).

[8] C. W. Tang, Appl. Phys. Lett. 48, 183 (1986).

[9] S. A. Van Slyke, C. H. Chen, and C. W. Tang, Appl. Phys. Lett. 69, 2160 (1996).

[10] J. Jeong, S. Park, S. J. Kang, H. Lee, and Y. Yi, J. Phys. Chem. C 120, 2292 (2016).

[11] A. Kumar, J. Brunet, C. Varenne, A. Ndiaye, A. Pauly, M. Penza, and M. Alvisi, Sens. Actuators B Chem. 210, 398 (2015).

[12] G. Sfyri, Q. Chen, Y.W. Lin, Y.L. Wang, E. Nouri, Z.X. Xu, and P. Lianos, Electrochim. Acta 212, 929 (2016).

[13] B. Wang, X. Zuo, Y. Wu, Z. Chen, C. He, and W. Duan, Sens. Actuators B Chem. 152, 191 (2011).

[14] T. Yu, Q. T. Zhang, D. P. Liu, and X. F. Han, Appl. Phys. Lett. 102, 022401 (2013).

[15] H. Hintz, H. J. Egelhaaf, H. Peisert, and T. Chassé, Polym. Degrad. Stabil. 95, 818 (2010).

[16] T. Nagata, S. Oh, T. Chikyow, and Y. Wakayama, Org. Electron. 12, 279 (2011).

[17] K. Jung, S. Park, Y. Lee, Y. Youn, H. I. Shin, H. K. Kim, H. Lee, and Y. Yi, Appl. Surf. Sci. 387, 625 (2016).

[18] J. Y. Kim, D. M. Lee, J. K. Kim, S. H. Yang, and J. M. Lee, Appl. Surf. Sci. 265, 145 (2013).

[19] H. Lee, J. Lee, K. Jeong, Y. Yi, J. H. Lee, J. W. Kim, and S. W. Cho, J. Phys. Chem. C 116, 13210 (2012).

[20] S. W. Cho, L. F. J. Piper, A. DeMasi, A. R. H. Preston, K. E. Smith, K. V. Chauhan, P. Sullivan, R. A. Hatton, and T. S. Jones, J. Phys. Chem. C 114, 1928 (2010).

[21] I. Biswas, H. Peisert, T. Schweiger, D. Dini, M. Hanack, M. Knupfer, T. Schmidt, and T. Chassé, J. Chem. Phys. 122, 064710 (2005).

[22] L. Ottaviano, L. Lozzi, F. Rispoli, and S. Santucci, Surf. Sci. 402404, 518 (1998). 